\title{
Reexposure to the amnestic agent alleviates cycloheximide-induced retrograde amnesia for reactivated and extinction memories
}

\author{
James F. Briggs ${ }^{1}$ and Brian P. Olson \\ Department of Psychology, Susquehanna University, Selinsgrove, Pennsylvania 17870, USA
}

\begin{abstract}
We investigated whether reexposure to an amnestic agent would reverse amnesia for extinction of learned fear similar to that of a reactivated memory. When cycloheximide $(\mathrm{CHX})$ was administered immediately after a brief cue-induced memory reactivation $(15 \mathrm{sec}$ ) and an extended extinction session $(12 \mathrm{~min})$ rats showed retrograde amnesia for both memories. CHX did not produce amnesia for a moderate extinction session $(6 \mathrm{~min})$. Re-administering $\mathrm{CHX}$ before testing reversed the amnestic effect for both memories (i.e., the memories were recovered). These results are discussed using a modified state dependent model of retrograde amnesia.
\end{abstract}

The consolidation theory proposes that memory formation of newly acquired information takes time to stabilize due to changes in neural processes, both synaptic and systemic. During this consolidation process, the memory is in a labile state making it susceptible to disruption until it becomes fully fixated (McGaugh 2000; Dudai 2004; see also Miller and Matzel 2006). Evidence for this is the time-dependent nature of retrograde amnesia (RA), i.e., forgetting events that occurred prior to the amnestic insult. It is well established that newly acquired memories are more vulnerable to disruption, while older well-consolidated memories are less likely to be affected (Ribot 1882; Duncan 1949; Mactutus et al. 1980; Judge and Quartermain 1982; for review, see Spear and Riccio 1994). This view describes memory loss as an interruption in storage, as older memories are "protected" from the amnestic insult because they have become fully consolidated.

A challenge to the consolidation view comes from findings that memories previously stored or consolidated could once again become labile and susceptible to disruption following reactivation (Misanin et al. 1968; Mactutus et al. 1979; Nader et al. 2000; for review, see Riccio et al. 2006). It is hypothesized that when a previously formed memory is reactivated by a reminder cue it goes through a reconsolidation process, which puts the memory in a labile state before again becoming consolidated. Thus, reactivating the memory with a reminder cue predisposes the memory to again be susceptible to an amnestic agent. These results suggest that the activity level is more important than the age of the memory in terms of vulnerability to an amnestic insult.

That old, well-established memories are once again vulnerable when activated challenges the storage failure view; however, other problematic evidence comes from findings that reexposure to the amnestic agent prior to testing alleviates RA (Thompson and Neely 1970; Hinderliter et al. 1975; Mactutus and Riccio 1978; Bradley and Galal 1988; Millin and Newman 2008). This reversibility of RA favors a retrieval failure view of forgetting, which suggests forgetting is due to the inability to access the information that has been stored. Accordingly, since the forgotten information can be recovered when the appropriate cues are provided just prior to test (i.e., reexposure to the amnestic agent), the information was presumably stored and the RA results from the inabil-

\section{${ }^{1}$ Corresponding author}

E-mail: briggsj@susqu.edu

Article is online at http://www.learnmem.org/cgi/doi/10.1101/Im.030270.113. ity to access the information because of the lack of appropriate retrieval cues (Spear 1978; Riccio et al. 2003, 2006).

This retrieval failure view has been described using a modified state dependent retention (MSDR) theory of RA first proposed by Hinderliter et al. (1975) (see also Millin et al. 2001; Riccio et al. 2003, 2006). Hinderliter et al. (1975) demonstrated that RA for a Pavlovian fear-conditioning task produced by hypothermia could be reversed by recooling the amnestic rats prior to testing. The results were explained by the MSDR view, which posits that when an amnestic agent (e.g., hypothermia) is administered during the processing or consolidation of the learned information, the information becomes encoded in the altered internal state (e.g., cool state). Thus, if tested in the "normal" state (normothermic) there would be a mismatch in cues and the memory would be inaccessible. However, reexposing the organism to the amnestic agent before testing returns the internal cues (i.e., cool state) which leads to the alleviation of RA.

The majority of research on the accounts of RA has been carried out using traditional learning paradigms; however, recent evidence suggests that RA for extinction of learned fear has similar characteristics to amnesia for original acquisition. In extinction, cues (conditioned stimuli) that have been previously paired with a biologically relevant reinforcer (unconditioned stimuli) are presented without the outcome, which leads to a reduction in responding. It is now well established that extinction involves new learning as evidence shows that the original response spontaneously recovers over time (Pavlov 1927) and that extinction learning is context specific (Bouton 1993, 2004). Extinction has also been shown to be susceptible to amnesia using a number of agents, including an NMDA antagonist (Falls et al. 1992), the protein synthesis inhibitors anisomycin (Berman and Dudai 2001; Vianna et al. 2001; Power et al. 2006) and CHX (Pedreira and Maldonado 2003; Sandusky et al. 2012), a sodium channel blocker (Eisenberg et al. 2003), ethanol (Lattal 2007), and hypothermia (Briggs and Riccio 2007). In addition to demonstrating RA for extinction, Briggs and Riccio (2007) also showed similarities in the characteristics of RA for original acquisition and extinction, such as the time dependent effect and alleviation of RA for extinction following reexposure to the amnestic agent.

The evidence above suggests that RA can be explained using a retrieval failure view and that RA for extinction shares similar characteristics as amnesia for original memories; however, it is not known whether amnesia for extinction using a protein synthesis 
inhibitor as the amnestic agent can be alleviated through reexposure. Protein synthesis inhibitors have long been used to induce amnesia, and have been shown to have a state dependency effect such that RA can be reversed by re-administering the agent prior to testing (Bradley and Galal 1988; Millin and Newman 2008). Thus, to facilitate the comparison of original acquisition memories and extinction memories, the aim of the current research was to investigate whether reexposure to the amnestic agent CHX would reverse RA for extinction, similar to that of an original acquisition memory.

In Experiment 1, we first sought to establish the exposure duration needed to observe the switch from RA for an old reactivated memory (i.e., reconsolidation) to RA for extinction using a passive avoidance procedure in adult female Long Evans rats. Both reconsolidation and extinction involve reexposure to previous conditioning cues, and thus an important question is: When does reconsolidation switch to extinction? Evidence suggests that the exposure duration is an important factor (Eisenberg et al. 2003; Dudai 2004; Suzuki et al. 2004; Power et al. 2006).

Training, extinction, and testing were conducted in a blackwhite shuttle box that was divided into two compartments. Following $2 \mathrm{~d}$ of handling, rats were randomly assigned to one of eight groups (see Table 1). Each group received punishment training. The rat was placed in the white side of the shuttle box with the door closed and then, after a brief period, the door was raised allowing the rat to cross to the black side. Once it entered the black side, the door was lowered and two inescapable footshocks ( $1 \mathrm{sec}, 0.5 \mathrm{~mA})$ were delivered. The interval between the two footshocks was $5 \mathrm{sec}$ and the animal was removed following the second footshock. This fear conditioning produces fear of the black compartment. An ANOVA revealed no differences in cross latencies at training between the eight groups $\left(F_{(7,56)}=\right.$ 0.76, $P>0.50$ ).

Twenty-four hours after being trained, six groups received a single extinction trial. In this session, rats were placed in the white compartment of the shuttle box for $15 \mathrm{sec}$, then in the black compartment for $15 \mathrm{sec}, 6 \mathrm{~min}$, or $12 \mathrm{~min}$. Rats were not able to cross between compartments, as the guillotine door was closed at all times. The parameters chosen for memory reactivation (15 sec) and extinction (12 min) were similar to those of previous studies (Hinderliter and Riccio 1977; Mactutus et al. 1979; Morgan and Riccio 1994; Harrod et al. 1996; Briggs and Riccio 2007). Two fear control groups did not receive extinction, but were merely tested to evaluate the level of fear without any reexposure treatment.

Following the extinction session, three experimental CHX groups received the amnesic treatment $(1.0 \mathrm{mg} / \mathrm{kg}$ i.p. CHX, premixed concentration of $0.1 \%$ [Oxoid Ltd.]) immediately following $15 \mathrm{sec}, 6 \mathrm{~min}$, or $12 \mathrm{~min}$ of extinction (15 s Ext, $6 \mathrm{~m} \mathrm{Ext}$, and $12 \mathrm{~m} \mathrm{Ext)}$ to assess RA for reactivated and extinction memories. Three saline control groups (15 s Ext, $6 \mathrm{~m}$ Ext, and $12 \mathrm{~m}$ Ext) received an equivalent volume $(1.0 \mathrm{mg} / \mathrm{kg})$ i.p. injection of

Table 1. Experimental design for Experiment 1

\begin{tabular}{|c|c|c|c|c|c|c|}
\hline Group & Training & $24 \mathrm{~h}$ & Extinction & Injection & $24 \mathrm{~h}$ & Test \\
\hline No Ext & Yes & & No & Saline & & Yes \\
\hline No Ext & Yes & & No & $\mathrm{CHX}$ & & Yes \\
\hline $15 \mathrm{~s}$ Ext & Yes & & $15 \mathrm{sec}$ & Saline & & Yes \\
\hline $15 \mathrm{~s}$ Ext & Yes & & $15 \mathrm{sec}$ & $\mathrm{CHX}$ & & Yes \\
\hline $6 \mathrm{~m} \mathrm{Ext}$ & Yes & & $6 \min$ & Saline & & Yes \\
\hline $6 \mathrm{~m} \mathrm{Ext}$ & Yes & & $6 \min$ & $\mathrm{CHX}$ & & Yes \\
\hline $12 \mathrm{~m} \mathrm{Ext}$ & Yes & & $12 \mathrm{~min}$ & Saline & & Yes \\
\hline $12 \mathrm{~m} \mathrm{Ext}$ & Yes & & $12 \mathrm{~min}$ & $\mathrm{CHX}$ & & Yes \\
\hline
\end{tabular}

(Ext) Extinction, (CHX) cycloheximide, $n=8$ for each group.

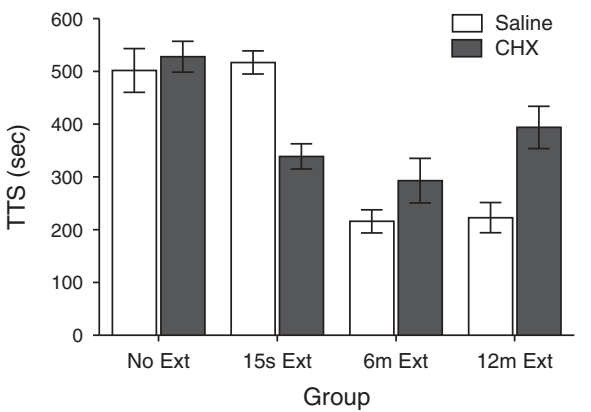

Figure 1. Mean ( \pm SEM) total time spent (TTS [in seconds]) on the safe (white) side of the black-white shuttle box for all groups in Experiment 1. (Open bars) Saline injection control groups, (shaded bars) CHX injected experimental groups. Long TTS scores for the No Ext control groups represent significant fear. Decreased TTS scores for the group that received $\mathrm{CHX}$ immediately following a brief reminder (15 s Ext, CHX) compared to the Saline group (15 s Ext, Saline) represent RA for a cue-induced reactivated memory. Increased TTS scores for the group that received CHX immediately after extinction ( $12 \mathrm{~m} \mathrm{Ext}, \mathrm{CHX}$ ) compared to the Saline group (12 m Ext, Saline) represents RA for extinction. Decreased TTS scores for the intermediate extinction Saline group ( $6 \mathrm{~m}$ Ext, Saline) compared to the No Ext control groups demonstrate sufficient extinction. Decreased TTS scores for the intermediate extinction CHX group (6 m Ext, CHX) compared to the No Ext control groups show the $\mathrm{CHX}$ had no effect on the RA for extinction.

physiological saline $(0.9 \%)$ immediately after extinction. The two fear control groups (No Ext) that did not receive extinction remained in their home cages and received injections of either $\mathrm{CHX}$ or saline $24 \mathrm{~h}$ after training.

Twenty-four hours after the extinction session, and $48 \mathrm{~h}$ after training for the fear control groups, all groups underwent passiveavoidance testing. Test trials were conducted identically to training trials with the rat starting in the white side of the shuttle box, except that no shocks were delivered upon entering the black compartment and the guillotine door remained open. Testing lasted for $10 \mathrm{~min}$ and the rat was allowed to choose between the white and black compartments. The total time spent on the safe (white) side was recorded as the dependent measure.

Figure 1 shows the mean total time spent on the safe side scores for all eight groups at testing. An ANOVA revealed that the groups differed significantly, $F_{(7,56)}=16.24, P<0.05$. Tukey HSD multiple comparison procedures were used to compare group differences. As can be seen, both No Ext groups had long test latencies and there were no differences between the Saline control group and the CHX only treatment group $(P>0.05)$. The groups that received the 15 -sec reminder followed by either saline or CHX did differ $(P<0.05)$, with the CHX group exhibiting less fear than the Saline group, demonstrating RA for an old reactivated memory. Groups that received $6 \mathrm{~min}$ of extinction did not differ $(P>$ $0.05)$. That the $6 \mathrm{~min}$ of extinction Saline group showed less fear and differed significantly from the No Ext groups $\left(P^{\prime}\right.$ s $\left.<0.05\right)$ illustrates the $6 \mathrm{~min}$ of exposure to the black side was sufficient to produce extinction. Interestingly though, there was no amnesia for extinction produced by the immediate injection of CHX, as the two $6 \mathrm{~m}$ Ext groups did not differ. These results are difficult to interpret since it is not clear whether the CHX again induced RA for the reconsolidation of the original memory or whether the amnestic agent had no effect on extinction. However, when the extinction session was extended to $12 \mathrm{~min}$ there were differences between the Saline and CHX groups $(P<0.05)$ with the CHX group expressing more fear, demonstrating that the protein synthesis inhibitor produced RA for extinction while leaving the original (reconsolidated) memory intact. 
Table 2. Experimental design for Experiment 2

\begin{tabular}{|c|c|c|c|c|c|c|c|}
\hline Group & Training & $24 \mathrm{~h}$ & Extinction & Injection & $24 \mathrm{~h}$ & Injection & Test \\
\hline No Ext/Sal & Yes & & No & Saline & & Saline & Yes \\
\hline No Ext/ReCHX & Yes & & No & $\mathrm{CHX}$ & & $\mathrm{CHX}$ & Yes \\
\hline $15 \mathrm{~s}$ Ext/Sal & Yes & & $15 \mathrm{sec}$ & Saline & & Saline & Yes \\
\hline $15 \mathrm{~s} \mathrm{Ext} / \mathrm{CHX}$ & Yes & & $15 \mathrm{sec}$ & $\mathrm{CHX}$ & & Saline & Yes \\
\hline $15 \mathrm{~s}$ Ext $/ \mathrm{ReCHX}$ & Yes & & $15 \mathrm{sec}$ & $\mathrm{CHX}$ & & $\mathrm{CHX}$ & Yes \\
\hline $12 \mathrm{~m} \mathrm{Ext} / \mathrm{Sal}$ & Yes & & $12 \mathrm{~min}$ & Saline & & Saline & Yes \\
\hline $12 \mathrm{~m} \mathrm{Ext} / \mathrm{CHX}$ & Yes & & $12 \min$ & $\mathrm{CHX}$ & & Saline & Yes \\
\hline $12 \mathrm{~m} \mathrm{Ext} / \mathrm{ReCHX}$ & Yes & & $12 \min$ & $\mathrm{CHX}$ & & $\mathrm{CHX}$ & Yes \\
\hline
\end{tabular}

(Ext) Extinction, (Sal) saline, (CHX) cycloheximide, (ReCHX) reexposure to $\mathrm{CHX} ; n=8$ for each group.

the 15 -sec reminder followed by either saline or CHX did differ $(P<0.05)$, with the CHX group exhibiting less fear, replicating RA for an old reactivated memory. Moreover, the groups that received $12 \mathrm{~min}$ of extinction followed by either saline or $\mathrm{CHX}$ were also significantly different $(P<0.05)$ with the $12 \mathrm{~m}$ Ext/CHX group showing more fear, which replicated RA for extinction. Importantly, the reactivated group (15s Ext ReCHX) and the extinction group (12 m Ext ReCHX) that received the second dose of $\mathrm{CHX}$ prior

These results show that CHX administered shortly after an old memory is activated can induce RA for the reactivated memory (disruption in reconsolidation). Moreover, the findings indicate that extinction is susceptible to RA produced by CHX, which is consistent with the concept that the extinction process is a form of new learning. When CHX was administered after an extended extinction session the original memory remained intact (reconsolidated) and amnesia for the extinction was observed. Thus, as others have shown, it appears that the exposure duration is an important factor for obtaining amnesia for extinction (Pedreira and Maldonado 2003; Suzuki et al. 2004; Power et al. 2006). That the original learning remained intact with longer exposure duration provides evidence that two memory traces must co-exist. Which trace becomes susceptible to disruption appears to be dependent on which process, i.e., reconsolidation or extinction, gains behavioral control (as pointed out by Eisenberg et al. 2003).

Experiment 2 served to determine whether RA for the reactivated memory and for extinction could be reversed by reexposing the rats to the amnestic agent, i.e., a second dose of CHX just prior to testing. All handling, training, extinction, injections, and testing were identical to those used in Experiment 1 except where noted. Following handling, rats were randomly assigned to one of eight groups (see Table 2) and all received training. An ANOVA revealed no differences in cross latencies at training among the eight groups $\left(F_{(7,56)}=0.54, P>0.50\right)$.

Twenty-four hours after training, three experimental groups were confined to the black compartment for $15 \mathrm{sec}$ (cue-induced reactivation; $15 \mathrm{~s}$ Ext). Three other experimental groups were confined to the black compartment for $12 \mathrm{~min}$ (extinction; $12 \mathrm{~m} \mathrm{Ext)}$ ). Of the six experimental groups, two groups from the reactivated condition (15 s Ext, Saline and CHX) and two from the extinction condition (12 m Ext, Saline and CHX) received injections $(1 \mathrm{mg} / \mathrm{kg}$ i.p.) of either saline or $\mathrm{CHX}$ immediately after being removed from the black compartment. Twenty minutes prior to testing, one group from each experimental condition (15 s Ext/ReCHX and $12 \mathrm{~m} \mathrm{Ext} / \mathrm{ReCHX}$ ) that received CHX following exposure received a second dose ( $1 \mathrm{mg} / \mathrm{kg}$ i.p.) of the protein synthesis inhibitor. All other experimental groups received saline injections ( $1 \mathrm{mg} / \mathrm{kg}$ i.p.) 20 min prior to testing. Two control groups (No Ext) received training, followed by an injection of either saline or CHX ( $1 \mathrm{mg} / \mathrm{kg}$ i.p.) $24 \mathrm{~h}$ after training, and received a second dose of saline or CHX $20 \mathrm{~min}$ prior to test. Testing for passive avoidance (fear of black compartment) was conducted $48 \mathrm{~h}$ after training.

Figure 2 shows the mean total time scores spent on the safe side for all eight groups at testing. An ANOVA revealed that the groups differed significantly, $F_{(7,56)}=8.78, P<0.05$. Tukey HSD multiple comparison procedures were again used to compare group differences. As can be seen, similar to the first experiment, both No Ext groups had long test latencies and there were no differences between the saline control group and the CHX-only treatment group $(P>0.05)$. In addition, the groups that received to test showed significant differences from their respective amnesia groups ( $P$ 's $<0.05$ ). Thus, the second dose of CHX before testing alleviated RA for both the cue-induced reactivated memory and the extinction memory. That the performance in the presence of CHX differed depending on which memory was affected rules out the recovery from amnesia being attributed to locomotor artifacts produced by the agent.

These novel results showing that the RAs observed for both a reactivated memory and an extinction memory are reversible by reexposing the rats to the amnestic agent $\mathrm{CHX}$ can be accounted for by using a retrieval failure view of RA that invokes a postextinction state dependent effect (Hinderliter et al. 1975; Millin et al. 2001; Riccio et al. 2003, 2006). According to this MSDR theory of RA, there is a short period after memory reactivation and extinction learning when the new information is processed. If the $\mathrm{CHX}$ is presented during processing, the information is encoded in an altered internal state produced by the agent. Thus, when later tested there is an encoding retrieval mismatch such that the appropriate internal retrieval cues are not available. However, if the $\mathrm{CHX}$ is re-administered before testing, the internal cues are restored, allowing appropriate retrieval of the memories.

Similar to reports using original acquisition memories (Bradley and Galal 1988; Millin and Newman 2008), RA for reactivated and extinction memories in the present studies is not produced by

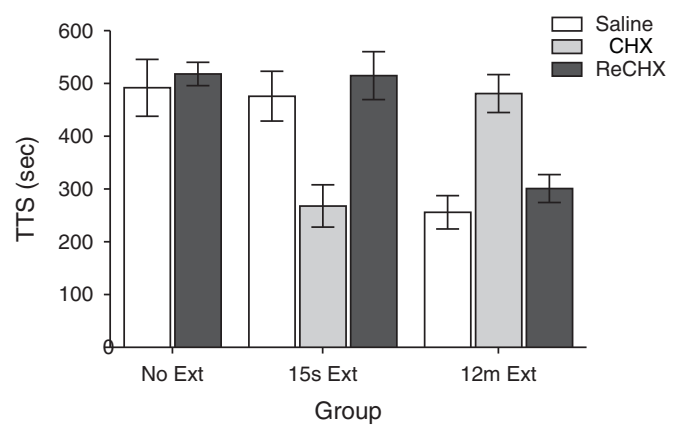

Figure 2. Mean ( \pm SEM) total time spent (TTS [in seconds]) on the safe (white) side of the black-white shuttle box for all groups in Experiment 2. (Open bars) Saline injection control groups, (light shaded bars) CHX injected experimental groups, (dark shaded bars) experimental groups that received a second $\mathrm{CHX}$ injection 20 min prior to testing. Long TTS scores for the No Ext control groups represent significant fear. A difference between the Saline and CHX groups for the $15 \mathrm{~s}$ Ext groups and the $12 \mathrm{~m}$ Ext groups represents a replication of RA for a reactivated memory and for extinction, respectively. Increased TTS scores for the group that was reexposed to the amnestic agent prior to test ( $15 \mathrm{~s}$ Ext, ReCHX) compared to the amnestic brief reminder group ( $15 \mathrm{~s}$ Ext, $\mathrm{CHX}$ ) demonstrates alleviation of RA for a cue-induced reactivated memory. Decreased TTS scores for the group reexposed to the CHX following amnesia for extinction (12 m Ext, ReCHX) compared to the amnesia for extinction group (12 m Ext, CHX) shows attenuation of RA for extinction. 
the inhibition of protein synthesis, but rather by a state dependent effect. This phenomenon raises an interesting question: Would similar results be obtained using site-specific manipulations? For example, Mamiya et al. (2009) recently demonstrated that infusing anisomycin directly into the hippocampus and amygdala produced amnesia for the reconsolidation of a contextual fear memory, while infusions directly into the amygdala and prefrontal cortex produced amnesia for extinction of contextual fear. According to the MSDR theory and the current results, the memory traces are formed but not accessible. Thus, although speculative, the amnesia produced by Mamiya et al. (2009) specific manipulations might be reversed by again infusing anisomycin before testing. This, of course, is an empirical question that requires further investigation.

In summary, the current findings contribute to our understanding that extinction as a form of new learning that shares similar characteristics to original acquisition memories by demonstrating that CHX induced RA for both a reactivated memory and for an extinction memory. The results that RA can be reversed for both the reactivated memory and for extinction by reexposure to the amnestic agent contribute to the literature on a retrieval-oriented account of RA using a modified state dependent explanation.

\section{Acknowledgments}

Partial funding of this research was provided by a Susquehanna University Committee on Faculty Scholarship grant to J.F.B. We thank Kendra Ashenfelder for helpful contributions to this project.

\section{References}

Berman DE, Dudai Y. 2001. Memory extinction, learning anew, and learning the new: Dissociations in the molecular machinery of learning in cortex. Science 291: 2417-2419.

Bouton ME. 1993. Context, time, and memory retrieval in the interference paradigms of Pavlovian learning. Psychol Bull 114: 80-99.

Bouton ME. 2004. Context and behavioral processes in extinction. Learn Mem 11: 485-494.

Bradley PM, Galal KM. 1988. State-dependent recall can be induced by protein synthesis inhibition: Behavioral and morphological observations. Dev Brain Res 40: 243-251.

Briggs JF, Riccio DC. 2007. Retrograde amnesia for extinction: Similarities with amnesia for original acquisition memories. Learn Behav 35: $131-140$.

Dudai Y. 2004. The neurobiology of consolidations, or, how stable is the engram? Annu Rev Psychol 55: 51-86.

Duncan CP. 1949. The retroactive effect of electroshock on learning. J Comp Physiol Psych 42: 32-44.

Eisenberg M, Kobilo T, Berman DE, Dudai Y. 2003. Stability of retrieved memory: Inverse correlation with trace dominance. Science 301: 1102-1104.

Falls WA, Miserendino MJD, Davis M. 1992. Extinction of fear-potentiated startle: Blockade by infusion of an NMDA antagonist into the amygdala. J Neurosci 12: 854-863.

Harrod SB, Metzger MM, Riccio DC. 1996. Does induced recovery from amnesia represent a disinhibition effect? Physiol Behav 60: 1375-1378.

Hinderliter CF, Riccio DC. 1977. Long-term effects of prior experience in attenuating amnesia. Am J Psychol 90: 407-418.

Hinderliter CF, Webster T, Riccio DC. 1975. Amnesia induced by hypothermia as a function of treatment-test interval and recooling in rats. Anim Learn Behav 3: 257-263.
Judge ME, Quartermain D. 1982. Characteristics of retrograde amnesia following reactivation of memory in mice. Physiol Behav 28: 585-590.

Lattal KM. 2007. Effects of ethanol on the encoding, consolidation, and expression of extinction following contextual fear conditioning. Behav Neurosci 121: 1280-1292.

Mactutus CF, Riccio DC. 1978. Hypothermia-induced retrograde amnesia: Role of body temperature in memory retrieval. Physiol Psychol 6: 18-22.

Mactutus CF, Riccio DC, Ferek JM. 1979. Retrograde amnesia for old (reactivated) memory: Some anomalous characteristics. Science 204: 1319-1320.

Mactutus CF, Ferek JM, Riccio DC. 1980. Amnesia induced by hyperthermia: An unusually profound, yet reversible, memory loss. Behav Neural Biol 30: 260-277.

Mamiya N, Fukushima H, Suzuki A, Matsuyama Z, Homma S, Frankland PW, Kida S. 2009. Brain region-specific gene expression activation required for reconsolidation and extinction of contextual fear memory. J Neurosci 29: 402-413.

McGaugh JL. 2000. Memory - a century of consolidation. Science 287: 248-251.

Miller RR, Matzel LD. 2006. Retrieval failure versus memory loss in experimental amnesia: Definitions and processes. Learn Mem 13: 491-497.

Millin PM, Newman EN. 2008. A comparison of the effects of state and non-state reminder treatments on morphine state-dependency and cycloheximide-induced retrograde amnesia in rats. J Behav Neurosci Res 6: $6-14$.

Millin PM, Moody EW, Riccio DC. 2001. Interpretations of retrograde amnesia: Old problems redux. Nat Rev Neurosci 2: 68-70.

Misanin JR, Miller RR, Lewis DJ. 1968. Retrograde amnesia produced by electroconvulsive shock after reactivation of a consolidated memory trace. Science 160: 554-555.

Morgan RE, Riccio DC. 1994. Extinction of an amnestic memory in rats: Evidence for the malleability of "inaccessible" information. Learn Motiv 25: $431-446$.

Nader K, Schafe GE, LeDoux JE. 2000. Fear memories require protein synthesis in the amygdala for reconsolidation after retrieval. Nature 406: $722-726$.

Pavlov IP. 1927. Conditioned reflexes: An investigation of the physiological activity of the cerebral cortex. Oxford University Press, London.

Pedreira ME, Maldonado H. 2003. Protein synthesis subserves reconsolidation or extinction depending on reminder duration. Neuron 38: $863-869$.

Power AE, Berlau DJ, McGaugh JL, Steward O. 2006. Anisomycin infused into the hippocampus fails to block "reconsolidation" but impairs extinction: The role of re-exposure duration. Learn Mem 13: 27-34.

Ribot TA. 1882. Diseases of memory: An essay in the positive psychology. Kegan Pay, Trench \& Co., London.

Riccio DC, Millin PM, Gisquet-Verrier P. 2003. Retrograde amnesia: Forgetting back. Curr Dir Psychol Sci 12: 41-44.

Riccio DC, Millin PM, Bogart AR. 2006. Reconsolidation: A brief history, a retrieval view, and some recent issues. Learn Mem 13: 536-544.

Sandusky LA, Flint RW Jr, McNay EC. 2012. Effects of the protein synthesis inhibitor cycloheximide on anxiety-like extinction behavior in an animal model of post-traumatic stress. Behav Brain Res 231: $208-212$.

Spear NE. 1978. The processing of memories: Forgetting and retention. Erlbaum, Hillsdale, NJ.

Spear NE, Riccio DC. 1994. Memory: Phenomena and principles. Allyn and Bacon, Boston, MA.

Suzuki A, Josselyn SA, Franklin PW, Masushige S, Silva AJ, Kida S. 2004. Memory reconsolidation and extinction have distinct temporal and biochemical signatures. J Neurosci 24: 4787-4795.

Thompson CI, Neely JE. 1970. Dissociated learning in rats produced by electroconvulsive shock. Physiol Behav 5: 783-786.

Vianna MRM, Szapiro G, McGaugh JL, Median JH, Izquierdo I. 2001. Retrieval of memory for fear-motivated training initiates extinction requiring protein synthesis in the rat hippocampus. Proc Natl Acad Sci 98: $12251-12254$.

Received January 4, 2013; accepted in revised form March 19, 2013. 


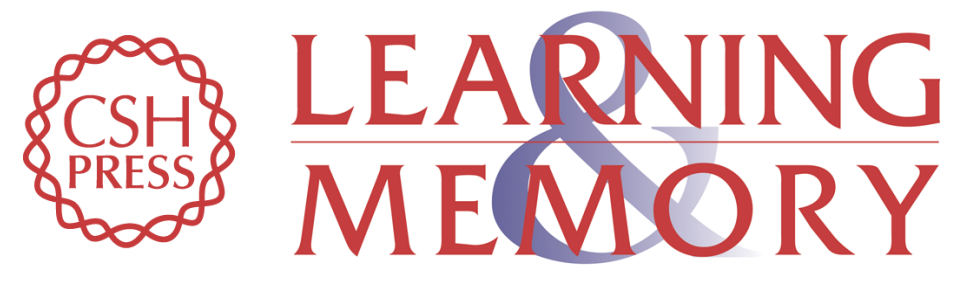

\section{Reexposure to the amnestic agent alleviates cycloheximide-induced retrograde amnesia for reactivated and extinction memories}

James F. Briggs and Brian P. Olson

Learn. Mem. 2013, 20:

Access the most recent version at doi:10.1101//m.030270.113

References This article cites 33 articles, 13 of which can be accessed free at: http://learnmem.cshlp.org/content/20/5/285.full.html\#ref-list-1

License

Email Alerting Receive free email alerts when new articles cite this article - sign up in the box at the Service top right corner of the article or click here. 